\title{
CALIDAD NUTRICIONAL Y CONSUMO DE MORERA (Morus alba), RAMIO (Bohemeria nivea (L) GAUD) Y SORGO NEGRO FORRAJERO (Sorghum almum) EN CABRAS1
}

\author{
Jorge Elizondo Salazar ${ }^{2}$
}

\begin{abstract}
RESUMEN
Calidad y consumo de morera (Morus alba), ramio (Bohemeria nivea (L) Gaud) y sorgo negro forrajero (Sorghum almum) en cabras. El experimento se llevó a cabo en la Estación Experimental Alfredo Volio Mata de la Universidad de Costa Rica. Se evaluó y comparó el consumo de morera, ramio y sorgo negro forrajero en nueve cabras raza La Mancha, con un peso promedio de $38( \pm 1) \mathrm{kg}$, distribuidas en un diseño de cuadrado latino 3 × 3 repetido. Los tratamientos experimentales fueron: a) dieta única de morera, b) dieta única de ramio y c) dieta única de sorgo negro. La morera con una edad de rebrote de 90 días, el ramio con 56 días y el sorgo negro con 70 días. El forraje se suministró en forma fresca picada (tallos y hojas). Se recolectaron muestras del material ofrecido y rechazado para el análisis de materia seca, proteína cruda y fibra neutro detergente. Se observaron diferencias altamente significativas $(\mathrm{P} \leq 0,01)$ para el consumo de forraje fresco, materia seca, proteína cruda y fibra neutro detergente. La ingesta de materia seca para los diferentes tratamientos se encontró dentro del rango reportado en la literatura, siendo de $1,94,0,97$ y 0,90 del peso vivo respectivamente. Con los consumos de proteína cruda obtenidos con la dieta de morera y ramio, los animales cubrieron sus necesidades de mantenimiento y suplieron el nutrimento de forma extra para alcanzar ganancias de peso diarias superiores a los 50 gramos. La dieta que presentó los mayores niveles de consumo de forraje verde, forraje seco, proteína cruda y fibra neutro detergente fue la correspondiente a morera, seguida de la dieta de ramio.
\end{abstract}

Palabras claves: cabras, Morus alba, sorgo negro forrajero, Sorghum almum, forrajes, Bohemeria nivea .

\begin{abstract}
Quality and intake of mulberry (Morus alba), ramie (Bohemeria nivea (L) Gaud) and black sorghum (Sorghum almum) fodder in goats. The experiment was carried out in the Experimental Station Alfredo Volio Mata of the University of Costa Rica. Mulberry, ramie and black sorghum fodder intake was evaluated and compared in nine LaMancha goats with an average body weight of $38( \pm 1) \mathrm{kg}$, distributed in a Latin square design $3 \times 3$ repeated. The experimental treatments were: a) mulberry fodder, b) ramie fodder and c) black sorghum fodder. Mulberry had 90 days, ramie 56 and black sorghum 70 days of re-growth. The forage was given as chopped materials (stems and leaves). Samples of offered and rejected material were gathered to analyze dry matter, crude protein and neutral detergent fiber content. Highly significant differences were observed $(\mathrm{P} \leq 0,01)$ for fresh forage, dry matter, crude protein and neutral detergent fiber intake by goats. Dry matter intake for the different treatments was within the range reported in the literature and they were $1,94,0,97$ and $0,90 \%$ of body weight for the different diets respectively. Crude protein intake with mulberry and ramie was high enough to cover maintenance requirements and they gave a surplus to obtain weight gains above 50 grams per day. Fresh forage, dry matter, crude protein and neutral detergent fiber intake were higher for mulberry followed by the ramie diet.
\end{abstract}

Key words: goats, Morus alba, black sorghum fodder, Sorghum almum, forages, Bohemeria nivea.

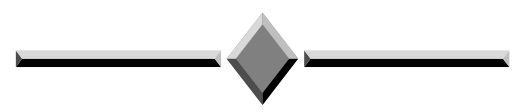

1 Recibido para publicación el 16 de abril del 2004. Inscrito en Vicerrectoría de Investigación de la Universidad de Costa Rica, Proyecto No. 737-98-005.

2 Estación Experimental Alfredo Volio Mata. Facultad de Ciencias Agroalimentarias. Universidad de Costa Rica. e-mail: jaelizon@cariari. ucr.ac.cr. Tel. (506) 279-5840. 


\section{INTRODUCCIÓN}

La gran aptitud de la cabra para la producción láctea, su facilidad de conversión alimenticia y sus altos índices de fertilidad y reproducción hacen que su explotación sea una alternativa para muchos productores a pequeña o gran escala, especialmente en regiones con escasos recursos forrajeros y con restricciones de área e instalaciones ya que por su pequeño tamaño y su capacidad para consumir diversas plantas disponibles le permiten adaptarse a una gran diversidad de medios con gran facilidad.

El hábito de ramoneo de la cabra, en la mayoría de los casos le permiten satisfacer sus necesidades alimenticias mejor que otras especies, en aquellos lugares en donde la disponibilidad de agua para producir forrajes es muy limitada (Agraz 1981). No por estas razones las cabras deben ser mal alimentadas, ya que los requerimientos nutricionales dependen de la etapa de vida en que se encuentren.

Al considerar la baja producción de leche por cabras en el trópico, puede decirse que se debe en parte al pobre mérito lechero de los animales, a deficiencias en el manejo, a la influencia del clima, pero principalmente a una deficiente alimentación (Devendra y Burns citados por Gutiérrez 1985). Es por esta razón que se debe buscar alternativas forrajeras que logren satisfacer las necesidades nutricionales de las cabras en todas sus etapas.

Existen en nuestro medio numerosas especies forrajeras y están disponibles en las fincas y presentan características de calidad nutritiva, disponibilidad, producción de biomasa y de versatilidad agronómica importantes, representando así un excelente potencial para mejorar la calidad alimenticia de las dietas de los animales e incrementar la producción. Entre ellas se encuentra la morera (Morus alba), perteneciente a la familia de las Moráceas.

La urgente necesidad de un forraje de alta calidad para alimentar rumiantes en el trópico y las excelentes características de la morera, son la justificación para el gran entusiasmo mostrado sobre su cultivo intensivo y uso como suplemento alimenticio en ganado mayor y alimento principal en cabras (Sánchez 2000).

En cuanto a su composición nutricional, Boschini (2002) encontró niveles promedios de materia seca de 23,7 y $23,6 \%$ en hojas y tallos respectivamente a los 84 días de rebrote y 22,22 y $7,75 \%$ de proteína cruda para esas mismas fracciones.

Otra especie que ha mostrado buenas características forrajeras es el ramio, perteneciente a la familia de las
Urticáceas. Elizondo y Boschini (2002) determinaron que a los 56 días de rebrote, la planta entera presentó un contenido de $14,56 \%$ de materia seca, $12,65 \%$ de proteína cruda y $50,63 \%$ de fibra neutro detergente.

Por otra parte, se encuentra el sorgo negro forrajero, perteneciente a la familia Poaceae. Su producción y calidad nutricional han sido reportados en diversas investigaciones (Amador y Boschini 2000, Hernández 1986 y Alfaro 1988), demostrando su excelente calidad para ser utilizado en la alimentación animal.

El presente trabajo se llevó a cabo con el fin de comparar la calidad, selección y consumo de morera, ramio y sorgo negro forrajero en cabras.

\section{MATERIALES Y MÉTODOS}

El experimento se llevó a cabo en la Estación Experimental de Ganado Lechero "Alfredo Volio Mata" de la Universidad de Costa Rica, ubicada a $1542 \mathrm{msnm}$, con una precipitación anual media de $2.050 \mathrm{~mm}$, distribuidos durante los meses de mayo a noviembre del 2002. La humedad relativa media es de $84 \%$ y la temperatura media de $19,5^{\circ} \mathrm{C}$.

La morera fue extraída de una plantación de 10 años, el sorgo negro de un cultivo con cuatro años y el ramio de un cultivo con tres años, todos en explotación continua. Los cultivos se dividieron en parcelas y se programó una uniformización escalonada cronológicamente para que la morera tuviera una edad de rebrote de 90 días, el ramio 56 días y el sorgo negro forrajero de 70 días. Todos los cultivos recibieron una fertilización nitrogenada equivalente a $150 \mathrm{~kg} / \mathrm{ha} /$ año de nitrógeno.

El forraje cosechado fue suministrado a nueve cabras de raza La Mancha no lactantes y no gestantes, con un peso vivo promedio de $38( \pm 1) \mathrm{kg}$, distribuidos en un diseño de cuadrado latino $3 \times 3$, repetido (Steel y Torrie 1988). Los tratamientos experimentales fueron los diferentes forrajes utilizados: a) dieta única de morera, b) dieta única de ramio y c) dieta única de sorgo negro. El forraje se ofreció a los animales en forma picada $(2,5$ $\mathrm{cm}$ ) tal como fueron cosechados (tallos y hojas). Cada animal fue ubicado en una jaula individual, recibiendo diariamente una relación de $14 \%$ de su peso vivo como forraje verde, en dos porciones iguales a las 7:00 a.m y a las 3:00 p.m. Cada período experimental fue de 28 días y previamente los animales tuvieron 14 días de adaptación a la nueva dieta. Cada animal se pesó al iniciar el ensayo y luego cada dos días hasta finalizar el experimento. El forraje rechazado se pesó diariamente a las 6:30 a.m. En días alternos se recolectaron muestras 
del material ofrecido y rechazado para ser enviado al laboratorio para su respectivo análisis. Los animales consumieron agua ad libitum. No se suplieron sales minerales durante el ensayo. En las muestras recogidas se analizó el contenido de materia seca, proteína cruda y fibra neutro detergente, mediante los procedimientos aprobados (AOAC 1980, Goering y Van Soest 1970) en cada caso.

Los datos obtenidos se analizaron con el PROC GLM del paquete estadístico SAS (1985). Las fuentes que resultaron estadísticamente diferentes, se sometieron a la prueba Duncan.

\section{RESULTADOS Y DISCUSIÓN}

El consumo y composición química de la morera, ramio y sorgo negro forrajero, se presentan en el Cuadro 1. Se encontraron diferencias altamente significativas $(\mathrm{P} \leq 0,01)$ en el consumo de forraje fresco (g/animal/día) entre los tratamientos, pudiéndose observar como la dieta de sorgo negro presentó el menor consumo por parte de los animales, mientras que la dieta de morera fue la que más consumieron, con una diferencia superior al $26 \%$ entre ambas.

Al cuantificar el consumo de forraje fresco como porcentaje del peso vivo del animal, puede observarse

Cuadro 1. Consumo y composición química de las dietas de morera, ramio y sorgo negro. Ochomogo, Cartago, Costa Rica. 2002.

\begin{tabular}{lrrr}
\hline \multicolumn{1}{c}{ Dieta } & Morera & Ramio & Sorgo negro \\
\hline $\begin{array}{l}\text { Forraje fresco } \\
\text { Consumido (g/día) }\end{array}$ & $3300,90 \mathrm{a}$ & $2745,00 \mathrm{~b}$ & $2610,00 \mathrm{c}$ \\
$\quad \begin{array}{c}\text { Consumido (\% } \\
\text { del peso vivo) }\end{array}$ & $8,69 \mathrm{a}$ & $7,22 \mathrm{~b}$ & $6,87 \mathrm{c}$ \\
& & & \\
Materia seca & & & \\
Ofrecida (\%) & $22,42 \mathrm{a}$ & $13,74 \mathrm{~b}$ & $12,98 \mathrm{c}$ \\
Consumida (\%) & $22,45 \mathrm{a}$ & $13,75 \mathrm{~b}$ & $13,69 \mathrm{~b}$ \\
Consumida (g/día) & $737,32 \mathrm{a}$ & $367,42 \mathrm{~b}$ & $342,03 \mathrm{c}$ \\
Consumida (\% & & & \\
del peso vivo) & $1,94 \mathrm{a}$ & $0,97 \mathrm{~b}$ & $0,90 \mathrm{c}$ \\
& & & \\
& & & \\
Proteína cruda & $13,96 \mathrm{a}$ & $17,58 \mathrm{~b}$ & $12,70 \mathrm{c}$ \\
Ofrecida (\%) & $15,71 \mathrm{a}$ & $20,57 \mathrm{~b}$ & $14,03 \mathrm{c}$ \\
Consumida (\%) & & & \\
Consumida (g/día) & $146,34 \mathrm{a}$ & $75,25 \mathrm{~b}$ & $48,50 \mathrm{c}$ \\
& & & \\
Fibra neutro detergente & & & \\
Ofrecida (\%) & $56,64 \mathrm{a}$ & $61,46 \mathrm{~b}$ & $69,56 \mathrm{c}$ \\
Consumida (\%) & $51,93 \mathrm{a}$ & $58,86 \mathrm{~b}$ & $68,45 \mathrm{c}$ \\
Consumida (g/día) & $382,88 \mathrm{a}$ & $209,24 \mathrm{~b}$ & $239,47 \mathrm{~b}$ \\
\hline
\end{tabular}

a, b, c Diferente letra entre dietas es significativo al $5 \%$. una relación de 8,69; 7,22 y 6,87\% para las dietas de morera, ramio y sorgo negro respectivamente.

Para el porcentaje de materia seca ofrecida, se encontraron diferencias altamente significativas $(\mathrm{P} \leq 0,01)$ entre los diferentes tratamientos. La dieta de sorgo negro presentó un contenido de $12,98 \%$, mientras que la dieta de morera fue superior en 9,44 puntos porcentuales, y el ramio de $13,74 \%$. El contenido de materia seca en el forraje consumido, igualmente varió significativamente $(\mathrm{P} \leq 0,01)$ entre tratamientos y fue mayor en el tratamiento de morera, llegando a ser de $22,45 \%$.

El consumo de materia seca con relación al peso vivo fue de 1,$94 ; 0,97$ y $0,90 \%$ para la dieta de morera, ramio y sorgo negro respectivamente. En este aspecto hay que considerar el amplio rango de valores que se reporta en la literatura, por ejemplo, Mc Cammon-Feldman $e t$ $a l$, citados por Sands (1983) revisaron la literatura relacionada con el consumo de materia seca por cabras y de 35 ensayos realizados en diferentes lugares del mundo, observaron que las cabras que consumieron gramíneas tropicales y/o plantas arbustivas, sin concentrado, tuvieron consumos entre 1,01 y $2,46 \%$ de su peso corporal. Por su parte, Medina (1992) obtuvo consumos promedios de materia seca de 1,94 y $0,92 \%$ del peso corporal para Guácimo (Guazuma ulmifolia) y pasto Guinea ( $\mathrm{Pa}$ nicum maximum) respectivamente. Oviedo et al (1995) reportan consumos de materia seca que varían entre 0,70 y $3,59 \%$ del peso corporal. Vallejo et al (1992) observaron consumos de materia seca con ensilaje de morera de 1,7\% del peso vivo. En otro estudio, Sahlu et al. (1992) obtuvieron consumos de materia seca entre 3,1 y $3,8 \%$ del peso vivo en cabras gestantes. Todos estos valores y rangos tan amplios, no dejan claro el panorama en cuanto al consumo de materia seca en cabras, sin embargo, es importante recalcar, que los altos niveles de consumo de materia seca que puedan alcanzar las cabras, no siempre son suficiente para garantizar un adecuado nivel nutricional, ya que lo más importante es cuantificar el consumo de nutrimentos.

El consumo diario (g/animal/día) de materia seca, proteína cruda y fibra neutro detergente, fue altamente diferenciado $(\mathrm{P} \leq 0,01)$ en las diferentes dietas. En la Figura 1, se observa el comportamiento en el consumo de las tres dietas estudiadas. Puede observarse claramente como el consumo de las tres fracciones fue mayor con la dieta de morera, mientras que los consumos más bajos de materia seca y proteína cruda se dieron con la dieta de sorgo.

El porcentaje de proteína cruda ofrecida y consumida tuvo diferencias altamente significativas $(P \leq 0,01)$ entre tratamientos. Puede notarse como el porcentaje de proteína cruda en el material ofrecido y consumido 


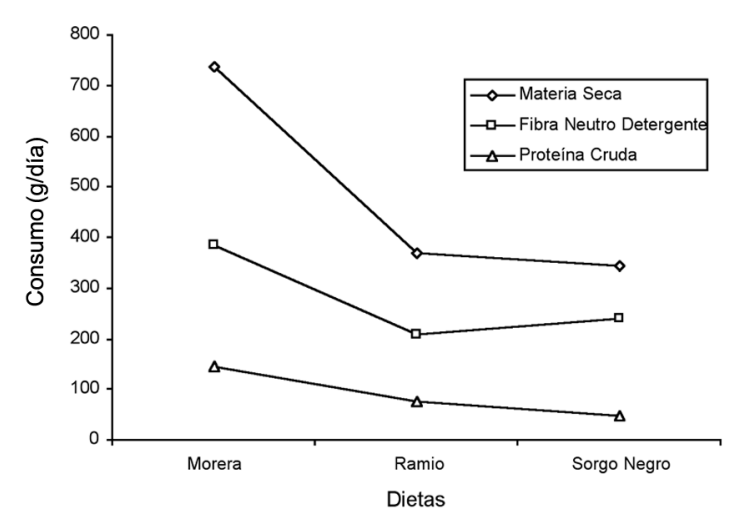

Figura 1. Consumo de nutrimentos en cabras, según las diferentes dietas. Ochomogo, Cartago, Costa Rica. 2002.

fue siempre superior en la dieta de ramio, no obstante debido al bajo consumo de materia seca con esta dieta, la cantidad de proteína cruda (gramos/animal/día) consumida no superó los 76 gramos.

La concentración de fibra neutro detergente en la materia seca ofrecida y consumida fue significativamente $(\mathrm{P} \leq 0,01)$ notoria entre los tres tratamientos estudiados. Este nutrimento fue siempre menor en la dieta de morera y mayor en la de sorgo.

Al analizar tanto la concentración de materia seca como la proteína cruda ofrecida y consumida, se encontró para ambos nutrimentos que lo consumido fue siempre superior a lo ofrecido, caso contrario sucede con la fibra neutro detergente, donde lo consumido fue siempre inferior a lo ofrecido. Esto demuestra la selección de las cabras aún cuando el material se ofreció en forma picada, ya que las cabras pueden compensar las situaciones particulares de baja calidad del forraje mediante el consumo preferencial de las fracciones vegetales de mayor calidad, en este caso las hojas, que es donde se concentra la mayor cantidad de materia seca y proteína cruda y menor cantidad de fibra.

Es importante resaltar que las cabras con mayores consumos de proteína cruda, tuvieron el mayor consumo de materia seca, mientras que aquellas que comieron menor proteína cruda tuvieron el consumo más bajo. Esto es consistente con Lu et al. (1990), quienes observaron que el nivel de proteína cruda en la dieta influye en el consumo voluntario de materia seca en cabras, pues un adecuado nivel de proteína en la dieta, propicia una mejor nutrición para los microorganismos del rumen.

La dieta que presentó el nivel más bajo de proteína cruda fue la de sorgo negro, con $12,70 \%$. Este valor no es bajo, si se compara con el nivel reportado por Min- son (1992) para las gramíneas forrajeras, que es inferior al $10 \%$, pero debido a los bajos consumos de materia seca obtenidos con esta dieta, los animales no llenaron sus requerimientos diarios de proteína cruda para mantenimiento que son de 58,66 gramos por animal por día (Elizondo 2002). Con las otras dos dietas experimentales, el consumo de proteína cruda fue superior a los requerimientos de mantenimiento. Para el caso de la dieta de ramio, éstos fueron superiores en un $28 \%$, mientras que para la dieta de morera fueron superiores en un $149 \%$. Al considerar la proteína cruda aportada por la dieta de morera, además de cubrir las necesidades de mantenimiento, suplió la proteína cruda necesaria para llenar los requerimientos de gestación. En caso de que fuera una cabra lactante, podría suplir la proteína necesaria para producir hasta 1,2 litros de leche con $4 \%$ de grasa. Si se considera la dieta de ramio, además de cubrir las necesidades de mantenimiento, puede suplir la proteína cruda necesaria para obtener ganancias diarias de peso superiores a los 50 gramos.

\section{CONCLUSIONES Y RECOMENDACIONES}

Las tres dietas evaluadas en el experimento, presentaron niveles inferiores al $22,42 \%$ de materia seca y niveles superiores al $12,5 \%$ de proteína cruda.

El consumo de sorgo negro forrajero fresco fue de $2610 \mathrm{~g} / \mathrm{animal} /$ día, lo que corresponde a un $6,87 \%$ de su peso vivo, mientras que el consumo de forraje seco fue de 342,03 gramos. Por otro lado, el consumo de ramio en fresco fue de $2.745 \mathrm{~g} / \mathrm{animal} /$ día, correspondiendo a un $7,22 \%$ del peso vivo de los animales. Finalmente, la dieta de sorgo negro forrajero fue la que presentó el menor consumo en fresco, siendo de $6,87 \%$ con relación al peso vivo del animal; mientras que la dieta de morera presentó el mayor consumo tanto en forraje verde como seco, siendo de $8,69 \%$ y $1,94 \%$ respectivamente.

Para las dietas de ramio y morera, los animales sobrepasaron los requerimientos de proteína cruda para mantenimiento, mientras que con la dieta de sorgo negro estuvieron 10 gramos por debajo.

La utilización de morera, ramio y sorgo negro forrajero en la alimentación de cabras, representan una buena alternativa, principalmente para ofrecer un forraje con un adecuado nivel de proteína cruda. Sin embargo, para el caso del sorgo negro es recomendable utilizarlo como complemento a una dieta rica en proteína, ya que como ingrediente único en la dieta, no permitiría a los animales cubrir los requerimientos de proteína cruda para mantenimiento. 
Una dieta única de ramio, llenaría las necesidades de proteína cruda para mantenimiento y además supliría las necesidades para ganancias de peso superiores a los 50 gramos diarios. Finalmente una dieta única de morera, permitiría cubrir los requerimientos de proteína cruda para mantenimiento y una producción diaria de leche estimada de 1,2 litros con $4 \%$ de grasa. Podría también llenar los requerimientos diarios de mantenimiento y gestación.

Debido al amplio rango de valores reportados en la literatura en lo referente a consumos de materia seca, es importante llevar a cabo ensayos que permitan cuantificar más adecuada y precisamente el consumo de nutrimentos por parte de los animales.

\section{LITERATURA CITADA}

AGRAZ, A. 1981. Cría y explotación de la cabra en América Latina. Editorial Hemisferio Sur S.A. Buenos Aires, Argentina. $481 \mathrm{p}$.

ALFARO, O. 1988. Evaluación de la productividad y calidad del sorgo negro forrajero (Sorghum almum) a través de diferentes distancias de siembra, densidades de siembra y niveles de fertilización nitrogenada. Tesis Ing. Agrónomo. UCR. San Pedro de Montes de Oca. 108 p.

AMADOR, A.; BOSCHINI, C. 2000. Calidad nutricional de la planta de sorgo negro forrajero (Sorghum almum) para alimentación animal. Agronomía Mesoamericana 11(2): 79-84.

A.O.A.C. (Association of Official Analysis Chemistry). 1980. Methods of Analysis. 13 th ed. Washington D.C. U.S.A. 168 p.

BOSCHINI, C. 2002. Nutritional quality of mulberry cultivated for ruminant feeding. In: Proceedings of an electronic conference carried out between May and August 2000. FAO. Roma. p. 171-180.

ELIZONDO, J. 2002. Estimación lineal de los requerimientos nutricionales del NRC para cabras. Agronomía Mesoamericana 13(2): 159-163.

ELIZONDO, J.; BOSCHINI. C. 2002. Calidad nutricional del ramio (Bohemeria nivea (L) Gaud) para alimentación animal. Agronomía Mesoamericana 13(2): 141-145.

GOERING, H.; VAN SOEST, P. 1970. Forage fiber analysis (Apparatus, reagents, procedures and some applications). Agricultural Handbook $\mathrm{N}^{\mathrm{o}} 379$. ARS-USDA, Washington, D.C. $76 \mathrm{p}$.
GUTIERREZ, R. 1985. Utilización del follaje de Poró (Eritrina poeppigiana) en combinación con banano (Musa sp. Cv. "Cavendish") como suplemento al pasto King Grass (Pennisetum purpureum $\mathrm{x}$ P. typhoides) en cabras lecheras estabuladas. Informe de trabajo especial para optar al grado de Mag. Sc, CATIE. Turrialba, Costa Rica. 15 p.

HERNÁNDEZ, E. 1986. Fertilización nitrogenada y edad de corte para sorgo negro (Sorghum almum). Tesis Ing. Agrónomo. UCR. San Pedro de Montes de Oca. 145 p.

LU, C.; POTCHOIBA, M.; SAÚL, T.; KAWAS, J. 1990. Performance of dairy goats fed soybean meal with or without urea during early lactation. Journal of Dairy Science (73): 726-734.

MEDINA, J. 1992. Observaciones sobre el consumo de follaje de Guácimo (Guazuma ulmifolia), Tiguilote (Cordia dentata) y pasto Guinea (Panicum maximum) por cabras semiestabuladas. In: Gutiérrez, F. (ed.). 1er Seminario Centroamericano de Agroforestería y Rumiantes Menores. Esquipulas, Chiquimula. Guatemala. p. 25-31.

OVIEDO, F.; BENAVIDES, J.; VALLEJO, M. 1995. Evaluación bioeconómica de un módulo agroforestal con cabras en el trópico húmedo. In: Benavides, J. (ed). Sistemas tradicionales y agroforestales de producción caprina en América Central y República Dominicana. CATIE. Turrialba, Costa Rica. p. 211-239.

SAHLU, T.; FERNANDEZ, J.; LU, C.; POTCHOIBA, M. 1992. Influence of dietary protein performance of dairy goats during pregnancy. Journal of Dairy Science (75): $220-227$

SANCHEZ, M. 2002. World distribution and utilization of mulberry and its potential for animal feeding. In: Sánchez, M. Proceedings of an electronic conference. FAO. Roma. 1-10 pp.

SANDS, M. 1983. Consumo de arbustos por los carpinos. In: Benavides, J. (ed.). Memorias del Taller Internacional sobre producción caprina. CATIE. Turrialba, Costa Rica. pp. 43-49.

SAS. 1985. Statistical analysis system. SAS User's Guide; Statistics (Versión 5 Ed.). SAS Institute Inc.Cary, NC. 373 p.

STEEL, R.; TORRIE, J. 1988. Bioestadística: principios y procedimientos. Mc Graw Hill. México, D.F. 633 p.

VALLEJO, M.; BENAVIDES, J.; ESQUIVEL, J. 1992. Observaciones sobre el consumo de ensilaje de follaje de árboles y arbustos por cabras. In: Gutiérrez, J. (ed.). I Seminario Centroamericano de Agroforestería y Rumiantes Menores. Chiquimulas, Guatemala. 14-20 pp. 